\title{
Toward a Platform for Structured Data Acquisition in Oncology: A Pilot Study on Prostate Cancer Screening
}

\author{
Maurice Henkela,b,c Kirsten D. Mertz ${ }^{b}$ Jonas Laux ${ }^{a}$ Matthias Klan ${ }^{a}$ \\ Christian Breit $^{c}$ Katharina Marston ${ }^{d}$ Marc O. Matthias ${ }^{\mathrm{e}}$ Sarah G. Dugas $^{\mathrm{e}}$ \\ Sebastian Manneck ${ }^{c}$ Bram Stieltjes ${ }^{a, c}$ Helge Seifert $^{\mathrm{e}}$ Christian Wetterauer $^{\mathrm{e}}$ \\ aResearch \& Analytic Services University Hospital Basel, Basel, Switzerland; 'b Institute of Pathology, Kantonsspital \\ Baselland, Liestal, Switzerland; ' Institute of Radiology, University Hospital Basel, Basel, Switzerland; 'Institute of \\ Pathology, University Hospital Basel, Basel, Switzerland; eInstitute of Urology, University Hospital Basel, Basel, \\ Switzerland
}

\section{Keywords \\ Data acquisition - Data integration - Data element · \\ Synoptic reporting $\cdot$ Clinical decision support system}

\begin{abstract}
Introduction: Physicians spend an ever-rising amount of time to collect relevant information from highly variable medical reports and integrate them into the patient's health condition. Objectives: We compared synoptic reporting based on data elements to narrative reporting in order to evaluate its capabilities to collect and integrate clinical information. Methods: We developed a novel system to align medical reporting to data integration requirements and tested it in prostate cancer screening. We compared expenditure of time, data quality, and user satisfaction for data acquisition, integration, and evaluation. Results: In a total of 26 sessions, 2 urologists, 2 radiologists, and 2 pathologists conducted the diagnostic work-up for prostate cancer screening with both narrative reporting and the novel system. The novel system led to a significantly reduced time for collection and integration of patient information $(91 \%, p<0.001)$, reporting in radiology $(44 \%, p<0.001)$ and pathology $(33 \%, p=0.154)$. The system usage showed a high positive effect on evaluated data
\end{abstract}

karger@karger.com www.karger.com/ocl

Karger!"
(C) 2021 The Author(s)

Published by S. Karger AG, Basel

This is an Open Access article licensed under the Creative Commons Attribution-NonCommercial-4.0 International License (CC BY-NC) (http://www.karger.com/Services/OpenAccessLicense), applicable to the online version of the article only. Usage and distribution for commercial purposes requires written permission. quality parameters completeness, format, understandability, as well as user satisfaction. Conclusion: This study provides evidence that synoptic reporting based on data elements is effectively reducing time for collection and integration of patient information. Further research is needed to assess the system's impact for different patient journeys.

(C) 2021 The Author(s).

Published by S. Karger AG, Basel

\section{Introduction}

An aging society with multimorbid patients requires an interdisciplinary management that is able to evaluate an ever-increasing amount of data. This evaluation includes collecting, cleaning, mapping, and transforming data in accordance with the intended purpose and finally the provision of meaningful information [1]. In patient care, multiple clinical departments acquire a variety of data which are stored in various formats and IT systems. Summarizing and presenting all of this information in a consistent and easily retrievable manner are a key component to gain a holistic understanding of the patient's health status and facilitates communication between treating physicians [2]. 
The heterogeneity of data in terms of syntax (e.g., free text notes, images, and laboratory values), format (e.g., PDF and DICOM), and semantics (e.g., meanings or interpretations) complicates data integration in healthcare [3-5]. Missing documentation standards in healthcare cause patient data to be highly variable and patient records in 1 hospital to look different from data of similar patients receiving similar treatment in another hospital [6]. As a result, physicians and researchers are faced with considerable challenges to integrate disparate data from various sources [7].

A promising approach to improve information quality in medical reports is synoptic reporting [8]. Synoptic reporting is a clinical documentation method that unifies data entry with defined data elements. Data elements are units of information that have a precise name, definition, and representation terms [9]. Instead of the current standard of highly variable narrative reports, information is entered in a standardized manner. This way of documentation is suitable for different purposes like medical history, radiological, and histological examinations, and it has already been shown that such reports are more accurate, clearer, and coherent than narrative reports [10]. The broadest application of synoptic reporting is currently in oncologic pathology [11-16], radiology [17, 18], and operative reports in surgery $[19,20]$. An essential prerequisite for the successful expansion of this reporting method is the development of international standards based on the best available evidence. Pathology, as the discipline with the longest experience in the application is driving the development of international standards in synoptic reporting. The International Collaboration on Cancer Reporting and the College of American Pathologists have established international standardization processes for the development of synoptic reports that are based on the best available evidence [21]. Synoptic reporting based on data elements enables the reuse of information for multiple purposes. Information can be presented according to requirements of the respective recipient, like the referring physician, multidisciplinary team (MDTs) meetings, or clinical research [2]. In this study, we developed a novel system to align synoptic reporting to the data integration requirements and compared it with the current standard.

\section{Methods}

In order to gain a deep understanding of the requirements for data acquisition and integration at our institution, we focused, as an example, on the diagnostic work-up in prostate cancer screening. To objectively describe this case, we engaged in a set of work- shops together with Roche Healthcare Consulting. In these workshops, we mapped the information flow, stakeholders, resources, times, and standards along the patient's journey. Based on this process map, we designed our solution approach. Since we work in an academic setting, the use of data for research purposes was included in the system design.

\section{Diagnostic Work-Up in Prostate Cancer Screening}

This study was conducted at the University Hospital Basel, Switzerland. The diagnostic work-up of the screening program includes patient history, PSA, MRI, targeted biopsy, and the initial survey of SF36, IPSS, and NIH-CPSI as surveys conveying the baseline for patient-reported outcome. Patient history, MRI, and biopsy results are documented in a traditional narrative report in 3 different systems (CIS, LIS, and RIS). SF36, IPSS, and NIH-CPSI were recorded paper-based, scanned, and stored in electronic medical record in the CIS. PSA is either visible via the hospitals LIS or, in the case of external assessment, documented manually during anamnesis in the EMR. After all examinations were completed, the results were evaluated by a specialist in urology. Any detected prostate cancer was discussed in the interdisciplinary tumor board of the genitourinary cancer center at the University Hospital Basel. During the tumor board, there is no visualization of the examination results. All information is read out of the respective reports and only mentally merged. The result of this integration is not documented.

\section{From Retrospective Mapping to Prospective Standardization}

The novel system is an in-house development and consists of a frontend, backend, SQL database, NoSQL database, and RESTful API. The frontend provides an overview of all patients included in the screening program, forms for each documentation step, reporting engines for the MRI and biopsy report, a data integration tool, and a dashboard for clinical decision-making. The forms for data entry ensure that all information along the patient's journey is entered in a structured and consistent manner using data elements. Data elements are standardized information units that have a unique name, attributes, and predefined relations to make them comparable and support their exchange between information systems. The solution provides forms for each documentation step (SF36, SPSS, NIH-CPSI, medical history, PSA, MRI report, and biopsy report). The recorded data elements of prostate MRI are based on the Guidelines for Multiparametric Prostate Magnetic Resonance Imaging PI-RADS v2 and recommendations [22]. Figure 1 shows the data entry form for the MRI report.

The recorded data elements of the biopsy of the prostate are based on the International Society of Urological Pathology Consensus Conference [23]. Figure 2 shows the data entry from for the biopsy report. All recorded data elements are stored in centralized SQL and NoSQL databases. Patient identifying data, such as name and date of birth were stored in the SQL database. Nonpatient identifying data, such as survey and examination results were stored in the NoSQL database. This split between databases allowed fast and safe exchange of information for research requirements, like in a multicenter trial. A referencing system ensured fixed links between related information within an examination as well as predefined links between related information across examinations, which have to be validated by a specialist. The use of a NoSQL database enabled fast queries for both clinical needs that require all information from 1 patient and research needs that re- 


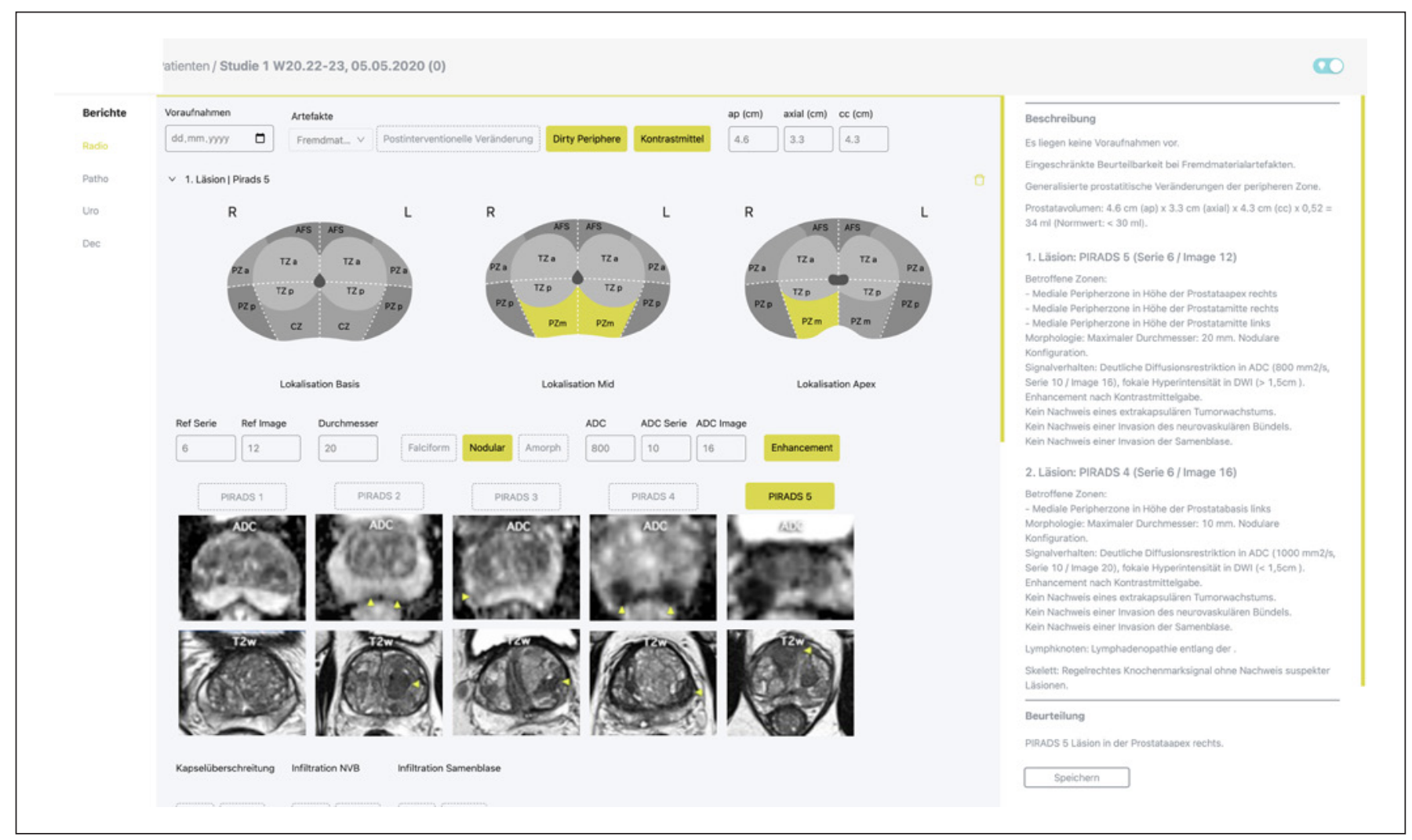

Fig. 1. Screenshot of the novel radiology user interface showing available reports (left), data entry form for the MRI report (middle) and automated report (right).

quire specific information from multiple patients with certain conditions. Queries of predefined research questions, for instance correlation between ADC values and Gleason scores, could also be executed web-based via the RESTful API.

The software provides summarized data in a patient overview that reduces manual data consolidation efforts. The patient overview displays how many examinations are completed along the patient's journey. Upon completion of all examinations along the diagnostic work-up, the data integration tool is available. Usually during MDTs, all examination results are reviewed in order to facilitate a treatment decision. The referencing system supports predefined links to integrate related information across examinations and departments, for example, assign MRI features of a tumor to its corresponding features form the biopsy. These context-specific links of functionally related information are integrated into the software to enable automated data evaluation (risk assessment, relevant statistics, and research questions) in real time. The case dashboard summarizes all information according to the needs of the decision-making process. To take full advantage of the system's ability to provide statistics and address research questions, predefined queries are provided in the backend.

Since traditional narrative reports represent the current standard for information exchange between physicians, the novel solution uses synoptic reporting to avoid additional effort for data acquisition in routine clinical workflow. The report engine consists of a library of standardized text modules that were compiled into a report according to a predefined set of rules. Depending on the data elements entered, the corresponding text modules are composed to a coherent report.

\section{Study Design}

To evaluate the novel solution for routine clinical use, it was compared to the current routine practice in regards of data quality, effort for data acquisition, integration, and evaluation. To reduce possible confounds due to case variations, each specialist, in a real clinical practice scenario, compiled the same set of cases for data acquisition, integration, and evaluation once using the current methods (narrative reporting) and once with the proposed solution. In order to avoid previous opinion bias, all cases were first compiled using the standard and afterward using the proposed solution. As in a similar previous study, a washout period of 4 weeks was instantiated between the sessions to reduce this bias [24]. Participants received training on how to use the solution before the actual investigation.

The solution was applied to the screening's entire diagnostic pathway from anamnesis in urology to the pathologically confirmed diagnosis. All departments involved in this pathway (urology, radiology, and pathology) contributed to the study. Each department provided 1 resident and 1 senior consultant to test the software. All users had several years of experience in narrative reporting and no prior experience in usage to the novel software. The 30 -min introduction to the novel software included finding and 


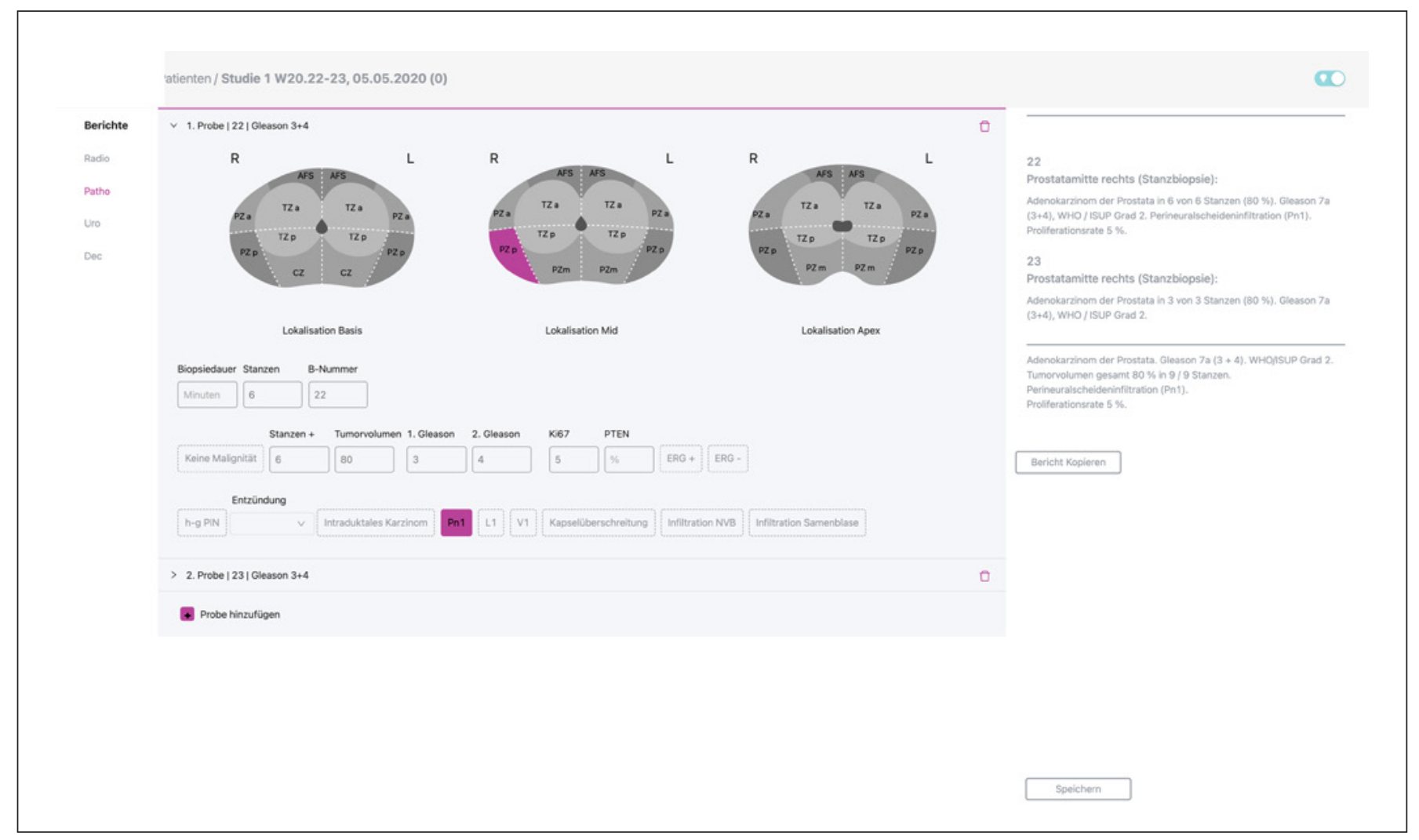

Fig. 2. Screenshot of the novel pathology user interface showing available reports (left), data entry form for the biopsy report (middle) and automated report (right).

opening a case, documentation of an exam, the report engine, the data integration tool, and the patient dashboard. For each case, the participants were instructed to perform their respective tasks in the screening process using the 2 methods (current vs. proposed solution). In both scenarios, the goal of a procedure recommendation after pathologically confirmed diagnosis was the same.

During the development of the software, the value of MRI in the screening program was investigated. Since the patients in this screening program undergo all the examinations that were covered by our novel system, these patients were selected for testing the software. At the beginning of the evaluation, 13 patients completed the screening's entire pathway to the pathologically confirmed diagnosis. All these cases were included in the evaluation, which resulted in 26 observation sessions reviewing the same twice. Characteristics of the selected cases are listed in Table 1.

The entire screening process was examined for expenditure of time, data quality, and user satisfaction. For each task, the time for both methods was recorded. Time per case was evaluated in total and separated for each specialty (urology, radiology, and pathology). Prior to the study, all necessary data elements for the treatment decision were defined. Based on the predefined data sets, both methods were evaluated for completeness. Furthermore, the perceived data quality was evaluation using a questionnaire for contextual information quality (completeness, relevance, timeliness, and usefulness) and representative information quality (for- mat, consistency, and comprehensibility). A questionnaire-based survey was used to evaluate a qualitative acceptance of the new system compared to the previous workflow [25]. The defined data elements and surveys are provided in Tables $2-5$.

\section{Statistical Analysis}

The Wilcoxon signed-rank test was used to compare case preparation time as a functional specialty (urology, radiology, and pathology) for the current method and the proposed solution. Cohen's $d$ was used to compare responses to the survey questions. $p<0.05$ was considered statistically significant. Analysis was conducted in $\mathrm{R}$ (R Core Team, 2014) and figures were produced using the package ggplot2 (Wickham [26]).

\section{Results}

\section{Turnaround Time}

The total turnaround time for the novel solution was statistically significantly lower than the current method $(-2.95 \mathrm{~min}, p<0.001)$. The paired analyses for turnaround times as a function of specialty showed statistically significant differences between the novel 
Table 1. Case characteristics

\begin{tabular}{|c|c|c|c|c|c|c|c|c|}
\hline 1 & 60 & NS & 6.80 & 30 & 5 & $7 \mathrm{a}$ & 2 & $\mathrm{RP} / \mathrm{RT}$ \\
\hline 2 & 73 & NS & 40.00 & 40 & 5 & $7 \mathrm{~b}$ & 2 & RP/RT \\
\hline 3 & 75 & NS & 3.99 & 22 & 4 & 6 & 1 & AS \\
\hline 4 & 74 & $\mathrm{~S}$ & 3.02 & 60 & 4 & $7 a$ & 2 & $\mathrm{RP} / \mathrm{RT}$ \\
\hline 5 & 61 & NS & 2.18 & 28 & 4 & 6 & 1 & AS \\
\hline 7 & 53 & NS & 2.88 & 23 & 4 & & & Follow-up \\
\hline 8 & 51 & NS & 10.10 & 64 & 3 & & & Follow-up \\
\hline 9 & 50 & NS & 0.41 & 20 & 4 & & & Follow-up \\
\hline 10 & 59 & $S$ & 0.43 & 15 & & & & Follow-up \\
\hline 11 & 73 & NS & 0.72 & 28 & 4 & & & Follow-up \\
\hline 12 & 75 & NS & 4.35 & 26 & 4 & HGPIN & & Re-biopsy \\
\hline
\end{tabular}

DRE, digital rectal exam; S, suspicious; NS, nonsuspicious; ISUP, International Society of Urological Pathology.

Table 2. Recorded data elements along the screening's pathway

\begin{tabular}{lll}
\hline Urology & Radiology & Pathology \\
\hline Family history & Date of old exams & Biopsy duration \\
Digital rectal examination & Artifacts & Number of punches \\
Previous surgery & Diameter ap & Number of punches with cancer \\
5 a-reductase inhibitor & Diameter axial & Tumor volume \\
Testosterone replacement therapy & Diameter cc & Gleason the most \\
Urinary tract infection & Contrast agents & Gleason the worst \\
PSA & Lesion description & Ki-67 \\
IPSS (1-8) & Reference series & PTEN \\
NIH-CPSI (1-9) & Reference image & ERG \\
SF36 (1-36) & Diameter & High-grade PIN \\
& Shape & Inflammation \\
& Localization & Intraductal carcinoma \\
& PI-RADS & Perineural invasion \\
& ADC & Vascular invasion \\
& Reference series & Lymphovascular invasion \\
& Reference image & Extraprostatic extension \\
& Contrast enhancement & Neurovascular bundles invasion \\
& Extraprostatic extension & Seminal vesicle invasion \\
& Neurovascular bundles invasion & \\
& Seminal vesicle invasion & \\
& Lymphnode invasion & \\
& Bone invasion &
\end{tabular}

IPSS, International Prostate Symptom Score; NIH-CPSI, National Institutes of Health-Chronic Prostatitis Symptom Index; SF36, short form (36) health survey; ADC, apparent diffusion coefficient; ap, anterior posterior; cc, craniocaudal; PTEN, phosphatase and tensin homolog; ERG, ETS-related gene; PIN, prostatic intraepithelial neoplasia. 
Table 3. Survey results of the effect size for comparison of contextual information quality with the current method and novel solution

\begin{tabular}{|c|c|c|c|}
\hline Construct & Item & Cohen's $d$ & $95 \% \mathrm{CI}$ \\
\hline \multirow[t]{2}{*}{ Completeness } & "The system provides me with complete information" & 0.69 & -0.63 to 2.01 \\
\hline & "The system produces comprehensive information" & 2.28 & $0.63-3.94$ \\
\hline \multirow[t]{3}{*}{ Relevance } & "Information within the system is applicable" & 2.33 & $0.67-4.00$ \\
\hline & "Information with the system is relevant for my job" & 0.55 & -0.76 to 1.86 \\
\hline & "In general, information within the system is relevant" & 0.73 & -0.60 to 2.06 \\
\hline \multirow[t]{2}{*}{ Timeliness } & The information provided within the system is up-to-date & 2.03 & $0.45-3.62$ \\
\hline & "The information provided within the system is received in a timely manner" & 3.05 & $1.16-4.94$ \\
\hline \multirow[t]{2}{*}{ Usefulness } & "Information within the system is informative" & 1.70 & $0.20-3.20$ \\
\hline & "Information within the system is valuable" & 1.85 & $0.31-3.39$ \\
\hline
\end{tabular}

Positive values indicate an effect in favor of the novel solution. Values above 0.2 reflect a low, above 0.5 a medium, and above 0.7 a high effect size.

Table 4. Survey results of the effect size for comparison of representational information quality with the current method using traditional reports and novel solution using charts

\begin{tabular}{|c|c|c|c|}
\hline Construct & Item & Cohen's $d$ & $95 \% \mathrm{CI}$ \\
\hline \multirow{2}{*}{ Format } & "The information provided within the system is well laid out" & 3.15 & $1.22-5.07$ \\
\hline & "The information provided within the system clearly presented on the screen" & 3.26 & $1.30-5.22$ \\
\hline \multirow[t]{2}{*}{ Consistency } & "Information within the system is accurate" & 2.08 & $0.48-3.68$ \\
\hline & "In general, information within the system is reliable" & 1.95 & $0.38-3.50$ \\
\hline \multirow[t]{2}{*}{ Understanding ability } & "Within the system, information is easy to comprehend" & 2.27 & $0.62-3.92$ \\
\hline & "Within the system, information that is clear in its meaning" & 2.26 & $0.61-3.91$ \\
\hline
\end{tabular}

Table 5. Survey results of the effect size for user satisfaction of the current method and the novel solution

\begin{tabular}{llrc}
\hline Construct & Item & Cohen's $d$ & 95\% CI \\
\hline Net benefits & "Using the system improves my content-related work" & 3.42 & $1.40-5.43$ \\
Workarounds & "Instead of using the system, I often use alternatives" & -0.50 & -1.80 to 0.81 \\
Satisfaction & "Overall, I am satisfied with the system" & 3.99 & $1.76-6.21$ \\
\hline
\end{tabular}

solution and the current method. Time for case evaluation in urology using novel solution decreased significantly compared to the current method $(-3.49$ $\min , p<0.001)$. Reporting time in radiology using the novel solution significantly decreased compared to the current method $(-3.29 \mathrm{~min}, p<0.001)$ and reporting time in pathology using the novel solution decreased compared to the current method $(-1.89 \mathrm{~min}$, $p=0.154)$. For a summary, please refer to Figures 3 and 4 and Table 6.

\section{Information Quality}

The objective evaluation for information completeness showed no missing information in either method. The results of the surveys on specific aspects of information quality are shown in Tables 3 and 4 . The effect size 


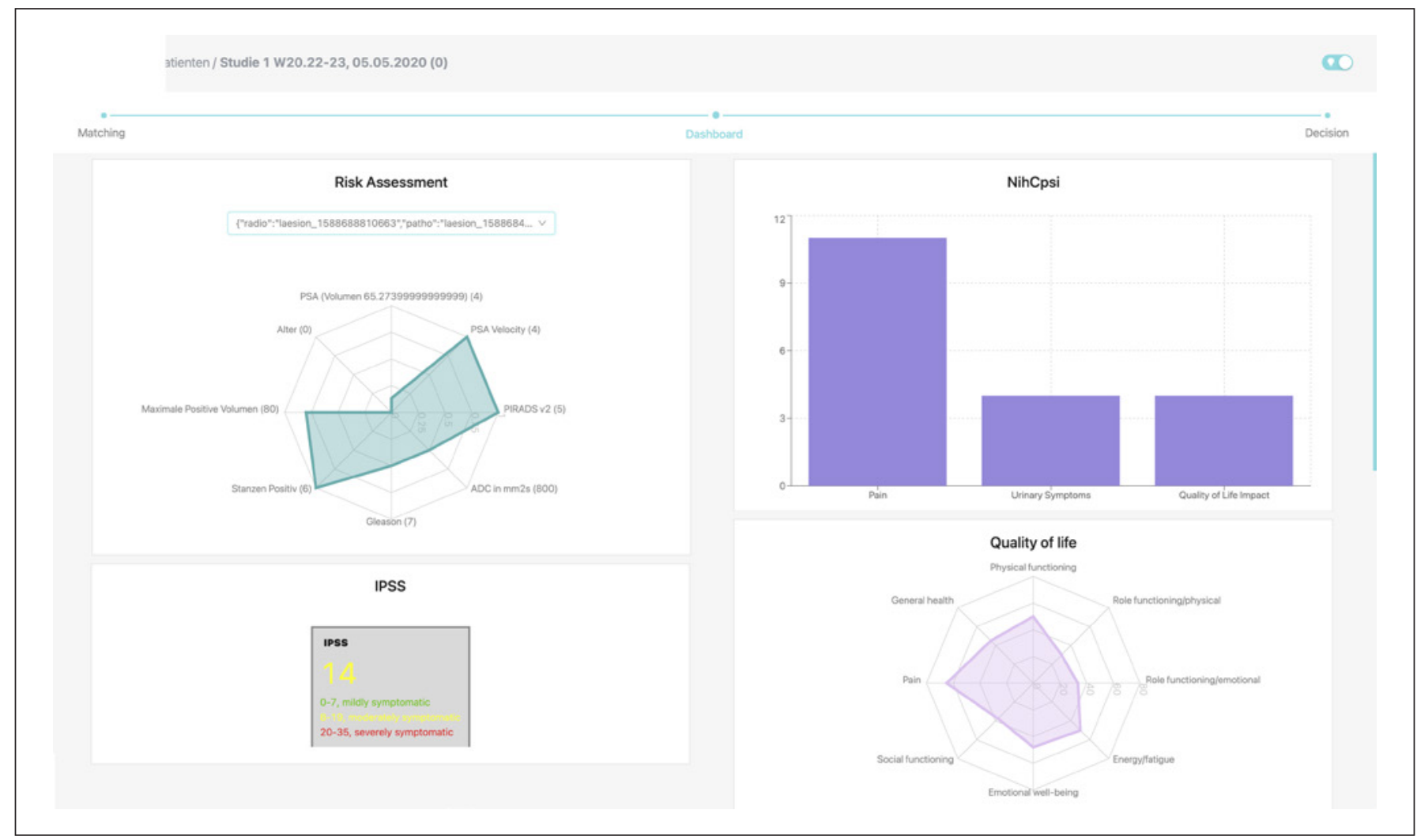

Fig. 3. Screenshot of the novel patient dashboard showing the available information in comprehensive charts. The risk assessment shows all information for each individual lesion (top left). NIH-CPSI (top right), quality of life (bottom left), and IPSS (bottom right) show results from the respective surveys.

Table 6. Mean and standard deviation values for total case turnaround times novel solution and the current method as a function of specialty

\begin{tabular}{|c|c|c|c|c|c|}
\hline \multirow[t]{2}{*}{ Specialty } & \multicolumn{3}{|c|}{ Mean (standard deviation) } & \multirow{2}{*}{$\begin{array}{l}\text { Difference, } \\
\%\end{array}$} & \multirow[t]{2}{*}{$p$ value } \\
\hline & novel (min) & current method (min) & difference (min) & & \\
\hline Pathology & $3.77(2.87)$ & $5.66(3.85)$ & -1.89 & $-33 \%$ & $=0.154$ \\
\hline Radiology & $4.18(1.22)$ & $7.47(3.08)$ & -3.29 & $-44 \%$ & $<0.001$ \\
\hline Urology & $0.36(0.17)$ & $3.85(1.09)$ & -3.49 & $-91 \%$ & $<0.001$ \\
\hline Total & $2.71(2.46)$ & $5.66(3.24)$ & -2.95 & $-52 \%$ & $<0.001$ \\
\hline
\end{tabular}

was calculated using Cohen's $d$. Positive values indicate an effect in favor of the novel solution. Values above 0.2 reflect a low, above 0.5 a medium, and above 0.7 a high effect size. The novel system demonstrated a high positive effect size on all evaluated dimensions of contextual and representational information quality. Content of information in the novel system was perceived to be more complete, relevant, up-to-date, and useful. Representation of information in the novel system was perceived to be more clear, consistent, and understandable.

\section{User Satisfaction}

The survey results indicated that use of the novel solution resulted in overall higher ratings on all of the ques- 


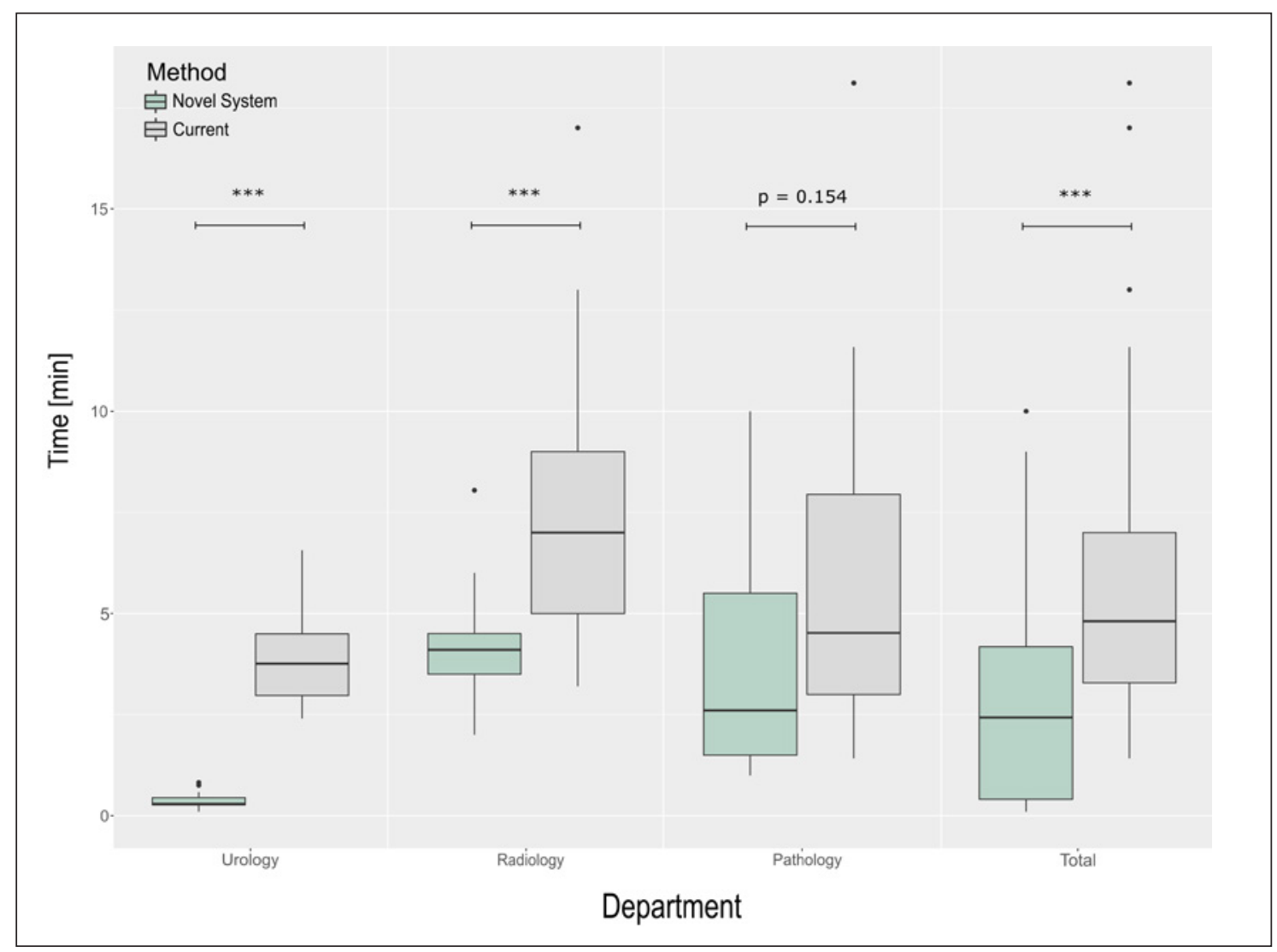

Fig. 4. Box plots for total case turnaround times novel solution and current methods as a function of specialty.

tions regarding ease of use and satisfaction. Synoptic reporting and the usage of charts for comprehensive information overview showed higher satisfaction, contributed to a statistically significantly improved patient evaluation, and reduced workarounds. Results for overall satisfaction across participants using Cohens's $d$ are shown in Table 5 .

\section{Discussion}

The integration of clinical information from multiple sources is essential to understand the patient's medical condition $[27,28]$. Missing documentation standards and siloed information management increase the effort for physicians to integrate patient information [29]. The alignment of medical reporting to data integration requirements can reduce physician's effort to understand the patient's medical condition and improve subsequent treatment decisions [30, 31].

In this study, we developed a novel system to align medical reporting to data integration requirements and compared it with the current standard. We evaluated the time required to report, review, and integrate patient data along the diagnostic work-up of prostate cancer screening. The usage of synoptic reporting reduced time for documentation of examination results compared to traditional narrative reporting, which is in line with previous studies [32]. Potential reasons include the reduced effort for text formatting $[32,33]$, correction of dictation errors (casus, numerus, and genus of word endings) [33], use of defaults (normal finding), automated calculations (prostate volume and tumor volumes in biopsies), proofreading for completeness, and consistency of the report [11, $13,14]$. The comparison of time savings between diagnostic departments showed greater time savings for radiology compared to pathology. This may be due to the longer reports in radiology, which allow for more time savings.

The urology department, which is responsible for review and integration of patient data, showed the greatest time savings of all departments. This can be explained by the usage of standardized definitions of information units 
and semantic relationships among these information units in an embedded referencing system. Information could thus be organized in a patient-centric manner, relieving urologists from the high manual effort of reviewing and integrating from multiple sources. In our study, data elements recorded along the diagnostic pathway were automatically prepared in a visually easily consumable way for the complex decision-making needs of MDTs. Since this presentation is not yet a routine procedure, no comparisons to the conventional approach with regard to time savings were made. However, previous studies were able to show that this way of organization and representation of information reduced case preparation time for decision-making processes [24, 34]. Given an aging society, the interdisciplinary management of multimorbid patients may lead to MDTs becoming primary recipients of examination reports. Therefore, MDT's requirements for information use should be better addressed in clinical reporting. Although not examined in this study, the patient is another important recipient of integrated data. Patients face enormous challenges in capturing, understanding, and acting on information from narrative reports. Data elements prepared in an easily consumable format, without technical terms, could provide patients with valuable insights about their health status and help them take a more active and empowered role in their care.

The survey results indicate that synoptic reporting improved data quality in terms of completeness, clarity, and consistency compared to narrative reporting. This is in line with previous studies and widely acknowledged in the diagnostic disciplines $[10,16,32,33,35$, 36]. Several studies demonstrated increased completeness in synoptical reports across various cancer reports including the breast, colorectal, lung, and prostate [35, 37-40]. User satisfaction in our study achieved significantly higher values than the current method. This is consistent with previous studies showing that the highlevel of clarity and consistency in synoptic reports leads to high satisfaction among oncologists, pathologists, and surgeons $[41,42]$. The optimized representation of patient information in comprehensive dashboards allows physician to focus on best treatment decisions rather than searching through poorly organized electronic medical records to retrieve relevant clinical information.

This study has the limitation of being confined to a single institution. This makes it difficult to generalize to other institutions using other IT systems. We also had limited ability to assess inter-user variability since only 2 participants were observed within each specialty. Nevertheless, the results based on 26 observation sessions represent a sufficient sample of observations. It is also limited by the fact that we only studied the linear pathway of prostate cancer screening. However, it is consistent with previous studies on synoptic reporting in different settings $[8,12-14,17,36,40,41]$. Our study can only serve as an initial assessment of the data integration requirements, as it does not reflect the full dynamics of the complex course of the disease.

\section{Conclusion}

Our study supports the hypothesis that synoptic reporting based on data elements is an effective method to reduce time for collection and integration of relevant patient information. Further work is needed to investigate the dynamics of complex courses of disease, multiple health conditions, and preparation of information in a suitable format for the patient.

\section{Acknowledgment}

We thank Ramon Höcker for the graphical input that improved the software.

\section{Statement of Ethics}

This study does not investigate diseases or the human body and is therefore not subject to the Human Research Act. The study is approved by the Ethikkommission Nordwest- und Zentralschweiz (January 14, 2020) and exempt from the Ethical Committee approval. All patient data used in this study were completely anonymized and are therefore not subject to the Data Protection Act. Informed consent of the patients is therefore not required.

\section{Conflict of Interest Statement}

The authors have no conflicts of interest to declare.

\section{Funding Sources}

Novartis Freenovation Award to B.S. 


\section{Author Contributions}

M.H., B.S., H.S., and C.W. determined system requirements. M.H., J.L., and M.K. developed the system. M.H. and B.S. determined the study design. K.D.M., C.B., K.M., S.G.D., S.M., and C.W. collected the data. M.H., K.D.M., C.W., and B.S. created the manuscript.

\section{Data Availability Statement}

All data generated or analyzed during this study are included in this article. Further enquiries can be directed to the corresponding author.

\section{References}

1 Lenzerini M, editor. Data integration: a theoretical perspective. Proceedings of the Twenty-First ACM SIGMOD-SIGACT-SIGART Symposium on Principles of Database Systems; 2002

2 Henkel M, Stieltjes B. Structured data acquisition in oncology. Oncology. 2020;98(6):4239.

3 Bansal SK, editor. Towards a semantic extract-transform-load (ETL) framework for big data integration. IEEE International Congress on Big Data; 2014.

4 Jayaratne M, Nallaperuma D, De Silva D, Alahakoon D, Devitt B, Webster KE, et al. A data integration platform for patient-centered ehealthcare and clinical decision support. Fut Gen Comput Syst. 2019;92:996-1008.

5 Barreto M, Denaxas S. Treating heterogeneity and uncertainty in data integration: study on Brazilian healthcare databases. Int J Population Data Sci. 2017;1.

6 Rajkomar A, Oren E, Chen K, Dai AM, Hajaj N, Hardt M, et al. Scalable and accurate deep learning with electronic health records. NPJ Digit Med. 2018;1(1):18.

7 Alshawi S, Missi F, Eldabi T. Healthcare information management: the integration of patients' data. Log Inform Manag. 2003;16:28695.

8 Baskovich BW, Allan RW. Web-based synoptic reporting for cancer checklists. J Pathol Inform. 2011;2:16.

9 Haacke EM, Duhaime AC, Gean AD, Riedy G, Wintermark M, Mukherjee P, et al. Common data elements in radiologic imaging of traumatic brain injury. J Magn Reson Imaging. 2010;32(3):516-43.

10 Słodkowska J, Cierniak S, Patera J, Kopik J, Baranowski W, Markiewicz T, et al. Functional assessment of synoptic pathology reporting for ovarian cancer. Pathobiology. 2016;83(23):70-8.

11 Donahoe L, Bennett S, Temple W, HilchiePye A, Dabbs K, Macintosh E, et al. Completeness of dictated operative reports in breast cancer: the case for synoptic reporting. J Surg Oncol. 2012;106(1):79-83.

12 Hassell L, Aldinger W, Moody C, Winters S, Gerlach K, Schwenn M, et al. Electronic capture and communication of synoptic cancer data elements from pathology reports: results of the Reporting Pathology Protocols 2 (RPP2) project. J Registry Manag. 2009;36(4): $117-5$; quiz 63-5.
13 Baranov NS, Nagtegaal ID, van Grieken NCT, Verhoeven RHA, Voorham QJM, Rosman C, et al. Synoptic reporting increases quality of upper gastrointestinal cancer pathology reports. Virchows Arch. 2019;475(2):255-9.

14 Gill AJ, Johns AL, Eckstein R, Samra JS, Kaufman A, Chang DK, et al. Synoptic reporting improves histopathological assessment of pancreatic resection specimens. Pathology. 2009;41(2):161-7.

15 Baskovich BW, Allan RW. Web-based synoptic reporting for cancer checklists. J Pathol Inform. 2011;2:16.

16 Mohanty SK, Piccoli AL, Devine LJ, Patel AA, William GC, Winters SB, et al. Synoptic tool for reporting of hematological and lymphoid neoplasms based on World Health Organization classification and College of American Pathologists checklist. BMC Cancer. 2007; 7(1):144.

17 Goel AK, DiLella D, Dotsikas G, Hilts M, Kwan D, Paxton L. Unlocking radiology reporting data: an implementation of synoptic radiology reporting in low-dose CT cancer screening. J Digit Imaging. 2019;32(6):104451.

18 Yeo I, Akwo J, Ekpo E. Automated mammographic density measurement using Quantra $a^{\mathrm{m}}$ : comparison with the Royal Australian and New Zealand College of Radiology synoptic scale. J Med Imaging. 2020;7(3):035501.

19 Cundy TP, Kirby CP, Kirby ML. Synoptic operative reports for quality improvement in pediatric cancer care. Pediatr Blood Cancer. 2018;65(10):e27238.

20 Brower ST, Katz M, Pisters P, Merchant N, Weber S, Posner M. Electronic synoptic operative reporting for pancreatic resection. J Am Coll Surg. 2011;212(3):425-6.

21 Hewer E. The oncologist's guide to synoptic reporting: a primer. Oncology. 2020;98(6): 396-402.

22 Weinreb JC, Barentsz JO, Choyke PL, Cornud F, Haider MA, Macura KJ, et al. PI-RADS Prostate Imaging - Reporting and Data System: 2015, Version 2. Eur Urol. 2016;69(1): $16-40$.

23 Epstein JI, Egevad L, Amin MB, Delahunt B, Srigley JR, Humphrey PA, et al. The 2014 International Society of Urological Pathology (ISUP) consensus conference on gleason grading of prostatic carcinoma: definition of grading patterns and proposal for a new grading system. Am J Surg Pathol. 2016;40(2): $244-52$.
24 Krupinski EA, Comas M, Gallego LG, Group G. A new software platform to improve multidisciplinary tumor board workflows and user satisfaction: a pilot study. J Pathol Inform. 2018;9:26.

25 Laumer S, Maier C, Weitzel T. Information quality, user satisfaction, and the manifestation of workarounds: a qualitative and quantitative study of enterprise content management system users. Eur J Inf Syst. 2017;26(4): 333-60.

26 Wickham H. ggplot2: elegant graphics for data analysis. Springer; 2016.

27 Kawamoto K, Houlihan CA, Balas EA, Lobach DF. Improving clinical practice using clinical decision support systems: a systematic review of trials to identify features critical to success. BMJ. 2005;330(7494):765.

28 Musen MA, Middleton B, Greenes RA. Clinical decision-support systems. Biomedical informatics. Springer; 2014. p. 643-74

29 Arndt BG, Beasley JW, Watkinson MD, Temte JL, Tuan WJ, Sinsky CA, et al. Tethered to the EHR: primary care physician workload assessment using EHR event log data and time-motion observations. Ann Fam Med. 2017;15(5):419-26.

30 Lankshear S, Srigley J, Mcgowan T, Yurcan M, Sawka C. Standardized synoptic cancer pathology reports - so what and who cares? A population-based satisfaction survey of 970 pathologists, surgeons, and oncologists. Arch Pathol Lab Med. 2013;137:1599-602.

31 Srigley J, Lankshear S, Brierley J, Mcgowan T, Divaris D, Yurcan M, et al. Closing the quality loop: facilitating improvement in oncology practice through timely access to clinical performance indicators. J Oncol Pract. 2013; 9:e255-61.

32 Qu Z, Ninan S, Almosa A, Chang KG, Kuruvilla S, Nguyen N. Synoptic reporting in tumor pathology: advantages of a web-based system. Am J Clin Pathol. 2007;127(6):898903.

33 Murari M, Pandey R. A synoptic reporting system for bone marrow aspiration and core biopsy specimens. Arch Pathol Lab Med. 2006;130(12):1825-9.

34 Fowler D, Sheets LR, Prime MS, Guo C, Siadimas A, Levy YZ, et al. Effect of digital tumor board solutions on "failure-to-discuss" rates for patient cases during tumor boards. JCO. 2019;37(27_Suppl 1):308. 
35 Sluijter CE, van Lonkhuijzen LR, van Slooten HJ, Nagtegaal ID, Overbeek LI. The effects of implementing synoptic pathology reporting in cancer diagnosis: a systematic review. Virchows Arch. 2016;468(6):639-49.

36 Srigley JR, McGowan T, Maclean A, Raby M, Ross J, Kramer S, et al. Standardized synoptic cancer pathology reporting: a populationbased approach. J Surg Oncol. 2009;99(8): $517-24$.

37 Messenger DE, McLeod RS, Kirsch R. What impact has the introduction of a synoptic report for rectal cancer had on reporting outcomes for specialist gastrointestinal and nongastrointestinal pathologists? Arch Pathol Lab Med. 2011;135(11):1471-5.
38 Haydu LE, Holt PE, Karim RZ, Madronio CM, Thompson JF, Armstrong BK, et al. Quality of histopathological reporting on melanoma and influence of use of a synoptic template. Histopathology. 2010;56(6):76874.

39 Karim RZ, Van Den Berg KS, Colman MH, McCarthy SW, Thompson JF, Scolyer RA. The advantage of using a synoptic pathology report format for cutaneous melanoma. Histopathology. 2008;52(2):130-8.

40 Aumann K, Amann D, Gumpp V, Hauschke D, Kayser G, May AM, et al. Template-based synoptic reports improve the quality of pathology reports of prostatectomy specimens. Histopathology. 2012;60(4):634-44.
41 Lankshear S, Srigley J, McGowan T, Yurcan M, Sawka C. Standardized synoptic cancer pathology reports-so what and who cares? A population-based satisfaction survey of 970 pathologists, surgeons, and oncologists. Arch Pathol Lab Med. 2013;137(11):1599-602.

42 Hammond EH, Flinner RL. Clinically relevant breast cancer reporting: using process measures to improve anatomic pathology reporting. Arch Pathol Lab Med. 1997;121(11): 1171. 